\title{
Evaluation of Specialty Lipids Based on Fish Oil and Capric Acid on Blood Lipid Chemistry Using Animal Models
}

\author{
Fayez Hamam ${ }^{1}$, Mayyas Al-Remawi², Abdel Rahman Nasr ${ }^{1}$ \\ ${ }^{1}$ Department of Pharmacology and Toxicology, College of Pharmacy, Taif University, Taif, KSA \\ ${ }^{2}$ Department of Pharmaceutics and Pharmaceutical Technology, College of Pharmacy, \\ Taif University, Taif, KSA \\ Email: $\underline{\text { fhamam@yahoo.com, }}$ ․hamam@tu.edu.sa
}

Received 15 February 2015; accepted 4 March 2015; published 9 March 2015

Copyright (C) 2015 by authors and Scientific Research Publishing Inc.

This work is licensed under the Creative Commons Attribution International License (CC BY). http://creativecommons.org/licenses/by/4.0/

(c) (†) Open Access

\section{Abstract}

This study aimed to produce new generation of fats or oils, known as specialty lipids, from enzymatic reaction of fish oil rich in 0mega-3 fatty acids, serving as a source of essential fatty acids, and a medium-chain fatty acid (Capric acid, C10:0), for rapid energy release, using self-designed bioreactor. The beneficial aspects of the resultant specialty lipids (SL) on blood lipid chemistry were evaluated using animal models. Serum lipids were analyzed for lipid profile. Animals in the three treatments: Group 1 fed with corn oil, Group 2 fed with a physical blend (PB) of fish oil (F0) and capric acid (CA); and Group 3 fed with specialty lipids (SL) showed a significant $(p<0.05)$ decrease in the level of triacylglycerols (TAG) after 60 days compared to day zero, but the highest decrease was noted for the corn oil (CO) fed animals. Analysis of differences among groups showed that a significant $(p<0.05)$ difference was noted between treatment groups $(2$ and 3$)$ and the reference group. The level of total cholesterol (TC) was considerably $(p<0.05)$ increased in animals fed with corn oil, whereas the PB and SL had no effect on TC. Blood lipid analysis for Group 1 (fed with corn oil) and Group 3 (fed with SL) showed significant increase $(p<0.05)$ in high-density lipoprotein cholesterol (HDL-c). Meantime, animals in Group 2 showed insignificant increase ( $p>$ 0.05 ) in HDL-c level after 60 days of treatment. Although SL caused an increase in HDL-c level but lower than CO. Animals in CO group showed insignificant increase $(p>0.05)$ in the level of LDL-c, whereas SL did not cause any change in LDL-c level. On the other hand, the level of low-density lipoprotein cholesterol (LDL-c) is considerably $(p<0.05)$ decreased in animals fed with PB. Results showed that the difference between PB and SL animals was insignificant $(p>0.05)$ concerning TAG, TC, and LDL-c levels, but the only difference between these two groups was in the HDL-c level. The weight of animals was increasing with time in animals fed $\mathrm{CO}$, while it was decreasing with time for animals fed PB and SL. The difference in percent body change between SL and CO was signifi-

\footnotetext{
"Corresponding author.
} 
cant $(p<0.05)$ after 8 weeks of treatment.

Keywords

Specialty Lipids, Animal Study, Packed Bed Bioreactor, Serum Lipid Profile

\section{Introduction}

Hypercholesterolemia is known as the main significant modifiable risk factor for the cardio vascular disease (CVD). Early diagnosis and treatment (diet and/or drug) can result in reduction of incidence of CVD. A clinical relationship has been recognized between hyperlipidemia and incidence of cardiac disease [1]. Genetic susceptibility, high level of serum cholesterol, and a decreased amount of high-density lipoprotein (HDL-cholesterol) are the major risk factors linked with coronary heart disease [2]. Cardiovascular diseases (CVD) are the major cause of morbidity and mortality among the Saudi population [3]. According to WHO (2008), cardiovascular disease resulted in death of 144 per 100,000 in KSA. Coronary heart disease (CHD) constitutes one of the major health problems in the kingdom [4]. The dollar cost of CVD management in Saudi Arabia is significantly high as reflected in a considerable percentage of frequent hospital admissions and bed occupancy due to CVD [5].

The prevalence of high-risk amounts of serum lipids was reported in many studies over the past two decades in different areas of Saudi Arabia [6]-[8]. For example, the prevalence of hypercholesterolemia (HC > $6.2 \mathrm{mmol} / \mathrm{L}$ ) among Saudi males was 7\%, while it was $8 \%$ for Saudi females [7]. Al-Shehri and his colleagues [6] found the prevalence of high-risk levels of serum lipids in school children in Riyadh. The prevalence of total cholesterol (TC), LDL-c, Triacylglyerol (TAG)/HDL-c were 32.7\%, 33.1\%, 34.1\% and 22.0\%, respectively. A cross sectional national epidemiological study indicated high prevalence of metabolic risk factors for cardiovascular disease among Saudi people, aged 30 - 64 [8]. In conclusion, CVD is a serious and fast growing health problem in Saudi Arabia. It is considered the leading reason of morbidity and mortality and this health problem should be addressed.

There is an increasing concern about the link between a high uptake of certain types of fatty acids (FA) or an inappropriate balance of the different fatty acids in the diet and certain disease conditions, such as heart diseases, obesity and tumor. This necessitates the need for new generations of fats or oils (SLs) that keep the physical, functional and organoleptic features of traditional lipids. In the mean time they also provide specific health benefits [9]. Specialty lipids (SL) are triacylglycerols (TAG) or phospholipids (PL) in which fatty acids (FA) are positioned at specific locations in the glycerol backbone of TAG. They can be synthesized using chemical or enzymatic procedures [10]. In this study, the enzymatic method was selected over the chemical one because the former has many advantages over the latter. For instance, it manufactures SL with a defined structure and requires mild temperatures without producing side products. It is energy efficient and purification of products is easy [11].

Oils from fish and other marine organisms can be changed to incorporate medium-chain FA (MCFA, 6-12 carbon atoms, saturated), serving as a source of fast energy release, and long-chain polyunsaturated (LC PUSFA, >12 carbon atoms), acting as a rich source of essential FA.

Recognition of the health advantages linked with the intake of sea foods ( $\omega 3$ or n-3 FA) is one of the major promising events in human nutrition and prevention of disease over the past thirty years. LC PUSFA display multifunctional role in enhancement of health and protection against disease in the human body. For example, the vital health functions of the Omega- 3 were the subject of extensive research studies as more than 14,000 scientific research works have investigated the health role of the Omega-3 FA from FO at all phases of human life [12]. The n-3 FA can be obtained from different sources, such as FO, flax seeds and algae. The n-3 FA, eicosapentaenoic acid (EPA, C20:5 n-3) and docosahexaenoic acid (DHA, C22:6 n-3), from FO will be chosen over other sources of the Omega-3 for the following reasons: Firstly, FO (Omega-3) constitutes more than 80\% (by volume) of the n-3 FA market [12]; Secondly, the human body preferred the Omega-3 fatty acids found in FO as they are most balanced and readily absorbed compared to those found in flax seeds and algae [12].

The omega-3 FA have attracted attention of many research groups across the globe because they possess many health benefits related to CVD [13]-[16], inflammation [17], immune response [17], diabetes [18] [19], hyper- 
tension [19] [20], and renal disorder (progressive IgA nephropathy) [21]. Moreover, the significance of the n-3 FA has increased as a potential clinical tool for CVD. Harris and Schacky [22] proposed the Omega-3 index (EPA and DHA content of the red blood cells) as a new risk factor for death from coronary heart disease (CHD). The researchers suggested that the Omega- 3 index of $\geq 8 \%$ was linked with highest protection against CHD, while an index of $\leq 4 \%$ resulted in least protection against CHD [22]. Furthermore, this index had many attributes, such as novelty, physiologically relevant, ease of modification, and independent. These characteristics render it as a significant clinical tool for CHD.

Pure medium-chain triacylglycerols (MCT) display unique structural and Physiological attributes [23] [24]. MCT provide less energy $(6.5-8.3 \mathrm{kcal} / \mathrm{g}$ ) than LCT $(9 \mathrm{kcal} / \mathrm{g})$. They are different from other lipids because they can be absorbed via the portal system without being hydrolyzed and reesterified because of their relative solubility in water. MCT do not require packaging onto chylomicron to be transferred to the cells and have a more quick $\beta$-oxidation to produce acetyl CoA [25]. Affinity of MCT to accumulate as body fat is lower than LCFA. They are independent on carnitine (an enzyme needed for carrying of FA across the inner mitochondrion membrane) to reach the matrix of the mitochondria. For the above mentioned reasons, absorption, transport and metabolism of MCFA is much easier than LCFA and, thus, MCFA were selected in this study to be incorporated into FO rich in n-3 FA [26]. To our knowledge, no attempts have been made to produce SL at a large-scale using a bioreactor or nutritionally assess them using animal models. In this study, a sufficient amount of new generation of fats or oils was produced for in vivo studies using a novel bioreactor packed with a biocatalyst. The effects of SL on blood lipid chemistry of experimental animals were investigated, including TAG, TC, HDL-c, and LDL-c. Furthermore, the effect of different dietary treatment was assessed on percent change in body mass.

\section{Materials and Methods}

FO, known as MEG-3 was obtained from Ocean Nutrition Canada (Halifax, Nova Scotia, Canada). The main Omega-3 FA (mg of $1000 \mathrm{mg}$ ) present in the MEG-3 were: EPA; 22:5n-3 (80 mg = $8 \mathrm{wt} \%$ ), DHA; 22:6n-3 (120 $\mathrm{mg}=12 \mathrm{wt} \%)$. Thus, the total percent of the Omega-3 FA is $20 \mathrm{wt} \%$. Decanoic acid, known as capric acid (C10:0; 98.0\%) and a commercial sn-1,3-specific lipase (Lipozyme IM 60) from Rhizopusmiehei were obtained from Sigma Chemical Co. (St. Louis, MO, USA). Kits for determination of TAG, TC, HDL-c and were purchased from Kashef Diagnostics (Jeddah, KSA).

\subsection{Apparatus}

The apparatus (Figure 1) consisted of one enzyme column, product and reactant containers, a circulating water bath, and a peristaltic pump. The column dimensions were exactly the same as we had in an earlier study [a 25-mm inner diameter (id), a 33.5-mm outer diameter (od), and a length of $162 \mathrm{~mm}$ ] [11]. In this study we chose the single bed model over the two-bed model for two main reasons. First, the single bed model predicted and confirmed a high percent of incorporation of CA into FO (31.7 mol\% for the single bed vs $22.7 \mathrm{~mol} \%$ for the double beds) [11]. Secondly, the coefficient of variation $\left(\mathrm{R}^{2}\right)$ of the one bed model was much better than the two beds model ( $R^{2}$ for one bed $=0.79$ compared to $R^{2}=0.54$ for the two column), suggesting that the model fit for the one bed was better than the two beds [11].

\subsection{Packing of the Column and Determination of the Column Void Fraction}

Details of the loading of the bed with the immobilized enzyme (24 g) and determination of the column void fraction was the same as described by Hamam and Budge (26). The primary objective of this research work was to assess the effects of SL on lipid blood chemistry using animal models, rather than testing the effects of process parameters on percent incorporation of capric acid into fish oil. Therefore, the optimal operational conditions that were selected in the present study were the same as described in an earlier study for one-bed bioreactor [11]. The optimal process variables were: substrate molar ratio of 2.70:1 (CA:FO); temperature $\left(55^{\circ} \mathrm{C}\right)$, and flow rate of reactants $(0.5 \mathrm{~mL} / \mathrm{min})$.

\subsection{In Vivo Feeding Studies}

Eighteen Wistar male rats aged 5 - 6 weeks old were randomly distributed into three groups; each group composed of six rats fed diets formulated from the SL, a physically-blended lipid containing oils (free FA and FO) 


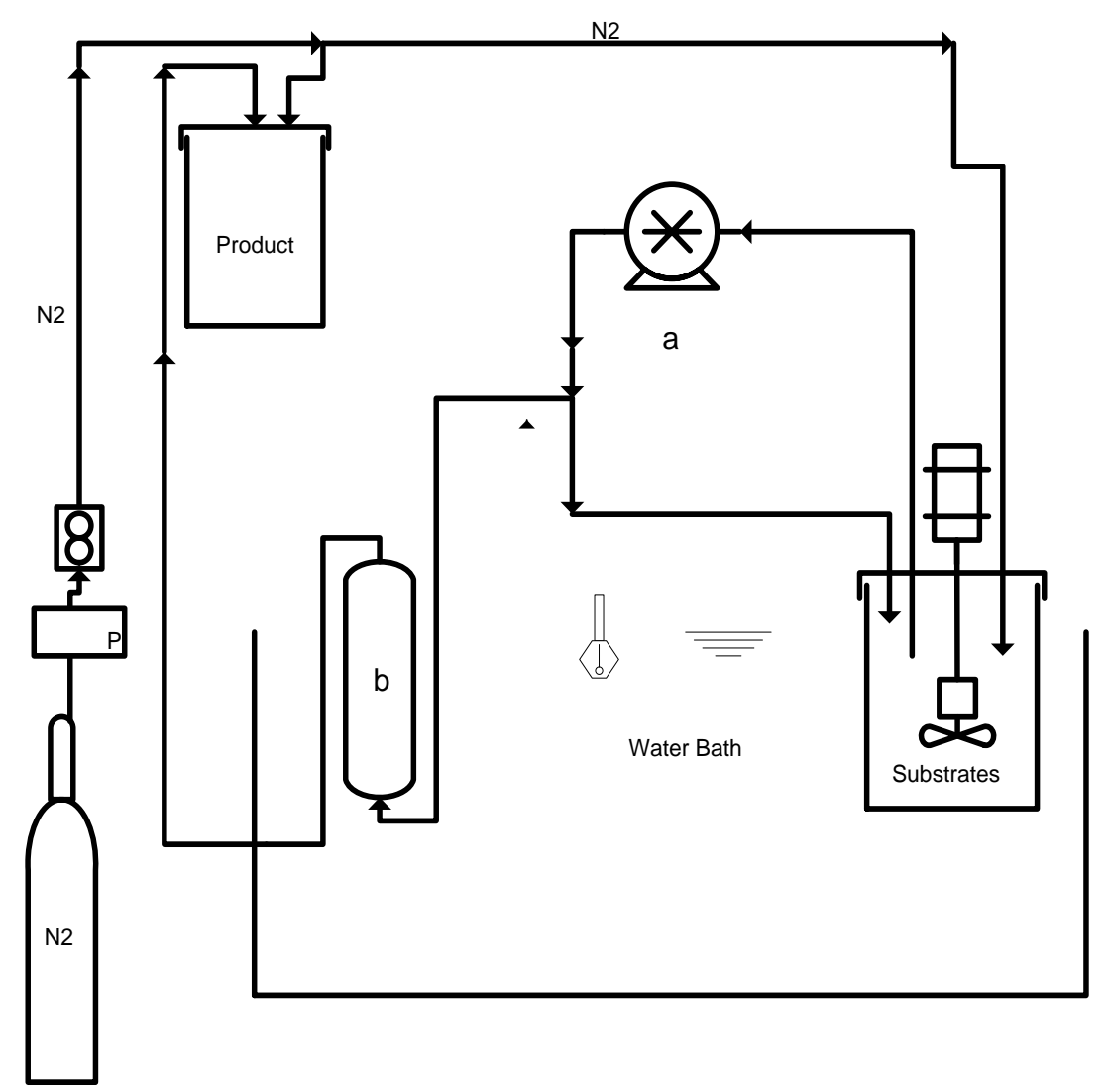

Figure 1. Schematic diagram of the single column bioreactor. a: peristaltic Master Flex pump (Cole-Parmer, Canada); b: enzyme bed packed with a sn-1,3 stereospecific immobilized lipase from Rhizomucormiehei (Lipozyme IM).

used to make the SL and a control lipid consisting of animals fed corn oil. The basal diets were based on standards used in other feeding studies, with each diet incorporating lipids at $10 \%$ of total diet mass. Animals have free access to food and water throughout the 60-day study period.

\subsection{Biochemical Testing of Serum Lipids}

Blood samples were collected at a specified time interval ( 0 and 60 days). Animals were fasted at least $16 \mathrm{~h}$ prior blood collection. A blood sample (2 - $3 \mathrm{ml}$ ) was withdrawn from each animal from the orbital sinus and collected into heparin treated microcentrifuge tubes at 0 and 60 days. Serum samples were prepared and stored at $-20^{\circ} \mathrm{C}$. Animal study of this work adhered to the European guidelines of ethics (EEC999 of Europe 1982). Serum lipids were collected and analyzed for lipid profile, including TAG, TC, HDL-c, and LDL-c by enzymatic methods using Khashef Diagnostic kits (Jeddah, KSA).

\subsubsection{Measurement of Serum Triacylglycerols (TAG)}

Level of triacylglyerols in the serum was measured using the glycerol phosphate oxidase (GPO) kit (Kashef Diagnostics, Jeddah, KSA). Briefly, serum TAG was hydrolyzed to glycerol and FFA by lipase, then glycerol was converted to glycerol-3-phosphate by glycerol kinase and ATP. The resultant glycerol-3-phosphate was oxidized by GPO to yield hydrogen peroxide, which produced a red colored dye in the presence of peroxidase, cholorophenol and 4-aminoantipyrine. The resultant red color product was measured spectrophotometrically at $500 \mathrm{~nm}$ using the UV-visible spectroscopy (UV-1800, Shimadzu, Kyoto, Japan). Level of serum TAG was calculated using Equation (1).

$[$ TAG $\mathrm{mg} / \mathrm{dL}]=[$ Abs sample $/$ Abs standard $] \times$ concentration standard 


\subsubsection{Measurement of Serum TC}

TC levels in the serum were determined after enzymatic hydrolysis and oxidation using cholesterol determination kit (Kashef Diagnostics, Jeddah, KSA). The resultant red color was quantified spectrophotometrically (UVvisible spectroscopy, UV-1800, Shimadzu, Kyoto, Japan) at $500 \mathrm{~nm}$. TC level was determined using the following equation:

$$
[\mathrm{TC} \mathrm{mg} / \mathrm{dL}]=[\text { Abs sample/Abs standard }] \times \text { concentration standard }
$$

\subsubsection{Measurement of Serum HDL-c}

Determination of HDL-c was performed using HDL-c determination kit (Kashef Diagnostics, Jeddah, KSA). HDL-c was separated from low density fractions (LDL- and VLDL-c) via precipitation with a mixture of phosphotungstic acid and magnesium chloride. HDL-c in the clear supernatant was measured according to the method described in Section 2.4.2.

\subsubsection{Measurement of Serum LDL-c}

Serum LDL-c level was determined using the Friedwald's method [27]. Determination of serum LDL-c amount was done after determination the level of TC, HDL-c and TAG. LDL-c level was calculated using the Friedwald's equation:

$$
\text { [LDL-cholesterol mg/dL] = TC }- \text { HDL-c }-[\text { TAG/5] }
$$

\section{Results and Discussion}

\subsection{Effects of CO, PB and SL Oil on Serum Triacylglycerol (TAG)}

The present study showed that TAG levels in Group 2 (fed with a PB of FO and CA) and Group 3 (fed SL) decreased level of TAG significantly $(p<0.05$ ) at Day 60 compared to Day 0 (Figure 2). Research works investigating the effects of CO on the serum TAG level using animal models are still producing conflicting results. Results of this study agree with many studies that indicated that CO resulted in a decrease in TAG level [28][30]. For example, Nalbone et al. [29] found that CO fed rats for eight weeks resulted in a significant decrease ( $40 \%)$ in TAG which was similar to our findings. However, Esmael and colleagues [30] showed that CO fed rats resulted in an increased level of TAG and LDL-c. Authors of this study explained one of the reasons for such increase could be due to an increase in the production and release of LDL-c and VLDL-c from the hepatic tissues onto the blood. The other possible reason could be related to increased enzyme activity that involved in the lipogensis. Another example that supports the idea that CO increased level of TAG came from the study that was conducted by Dauqan et al. [31]. Positive effects of CO on serum TAG could be explained as the following. Refined CO contains PUSFA (59\%), MUSFA (24\%) and SFA (13\%). Most of the PUSFA composed of linoleic acid (C18:2 n-6) and the ratio of n-6/n-3 reached a value of 83/1. Furthermore, it contains a substantial amount of antioxidants (ubiquinone and $\alpha$ - and $\gamma$-tocopherols) [32]. Thus, this fatty acid profile as well as the presence of antioxidants (e.g. ubiquinone and $\alpha$ - and $\gamma$-tocopherols) could decrease TAG level in rats.

Lee et al. [33] found that SL composed of soya bean and caprylic acid (C8:0) and the physical blend oil of soya bean and tricaprylin (C8:0/C8:0/C8:0) did not result in a significant difference in serum TAG, whereas results presented in this study showed that both PB and SL had a positive effects on serum TAG. Although rats fed SL showed lower serum TAG level than their counterparts who fed PB but this difference was statistically insignificant $(p>0.05)(95 \%$ CI $=-9.48$ - 22.16) (Table 1). All treatments (CO, PB, and SL) resulted in a decrease in TAG at Day 60, but the highest decrease in TAG was for the control oil group. When comparing TAG levels of PB and SL with TAG level in CO group at Day 60, it was obvious that both experimental groups showed significant difference $(p<0.05)(95 \%$ CI for CO and PB $=-34.31$ to -2.67 ; CI for CO and SL $=-40.66$ to -9.01$)$ (Table 1). Results of the present study agreed with those of Dauqan et al. [31] and Rezq et al. [34]. For instance, Dauqan et al. [31] found that PB of vegetable oils caused a considerable decrease in TAG levels in rats fed this mixture for 8 weeks. In this study and the previous one [11], the fish oil (MEG-3) used in the synthesis of SL contained a substantial amount of MUSFA ( 20\% mol\%) and much higher amount of PUSFA (32\% mol\%). This combination of MUSFA and PUSFA might promote a better clearance of TAG from the circulation. Since the sum (73\%) of MUSFA and PUSFA in CO is higher than its sum (52\%) in the PB or SL it would be expected that 


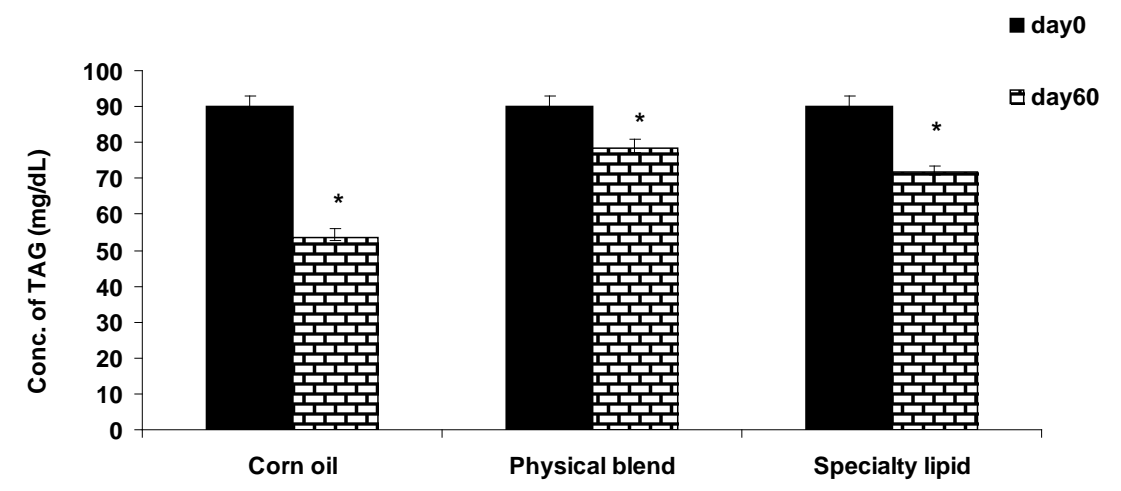

Figure 2. Triacylglycerol concentration (mg/dL) for corn oil, physical blend of fish oil and capric acid versus structured lipid composed of fish oil and capric acid at day zero and after 60 days of treatment. Error bars represent the standard error, ${ }^{*} p$-value $<0.05$ compared to day zero.

clearance of TAG from the circulation would be higher in rats fed with corn than their counterparts in the PB and SL groups. The other reason could be due to the presence of MCFA (C10:0), which exhibit unique structural and physiological features that make these fatty acids subjected to almost complete and faster hydrolysis, followed by fast transport to the liver via the portal system. Although fatty composition of the specific structured lipids and PB were the same, but results showed that SL has more effect on serum TAG at Day 60 compared to Day zero but this effect was insignificant $(p>0.05)$. The physical mixture and the SL could be absorbed in a different way because of their difference in stero-specific structures. The FA (MCFA and LCFA) are positioned equally at the three positions in the randomized TAG of a PB, whereas the MCFA are preferentially located at the terminal positions ( $s n-1$ and sn-3) in SL. Theoretically, serum TAG level in SL fed rats is expected to be lower than rat fed PB, when considering factors such as stereo-structures and composition and ratios of fatty acid. In conclusion, enhanced TAG clearance from the circulation, a better hydrolysis and absorption could be the main factors that resulted in such a decrease in TAG levels in rats fed the corn oil, physical blend and structured lipids.

\subsection{Effects of CO, PB and SL Oil on Serum Total Cholesterol (TC)}

Figure 3 showed the effect of different kinds of oils on serum TC level. The level of TC was considerably ( $p<$ 0.05 ) increased in animals fed with corn oil, which agrees with those of Ismael et al. [30]. The authors suggested that increased cholesterol level in rats fed CO might be attributed to high intake of exogenous cholesterol and subsequent deposition in the body. Another possible explanation could be due to a reduction in catabolism of cholesterol [30]. This assumption should be tested via examining level of bile acid as an indicator of decreased cholesterol catabolism. Others found different results [32]. For example, Dupont et al. [32] found that CO could act as a good cholesterol lowering agent because it contains high content of PUSFA (59\%) and low level of SFA (13\%). Furthermore, there are studies that tocotrienols could act as a cholesterol lowering agent via the suppression of cholesterol biosynthesis [35]. On the contrary, the PB resulted in a decrease in TC level, but this decrease was insignificant $(p>0.05)$ at Day 60 compared to Day 0 (Figure 3), but upon comparison with the animals fed CO, the level of TC was significantly lower $(p>0.05$, 95\% confidence interval CI $=5.42-45.01)$ at Day 60 (Table 1). FO contains a good amount of antioxidants (e.g. tocopherols) which several studies [35] showed that they could as a cholesterol lowering agent via inhibition of cholesterol biosynthesis. Thus, one of reasons to explain the reduction of cholesterol in PB group could be due to the presence of these antioxidants.

Rats fed SL for 60 days did not show any significant $(p>0.05)$ change in TC level compared to Day 0, but when compared to rats fed CO the level of TC was significantly lower $(p<0.05,95 \%$ CI $=13.05-52.64)$ (Table 1). Although, the TC levels among animals fed on PB and SL did not show any significant change ( $p>$ $0.05,95 \%$ CI $=-27.43-12.16$ ) (Table 1 ), but TC levels in both were considerably lower than the control group given CO. It is clear from these results that feeding SL rich in the omega-3 FA and CA or a physical mixture of FO and CA did result in an improvement of serum TC concentrations compared to the control corn oil group. The content of TC in the serum was not considerably different between the SL- and PB-fed animals, which 
Table 1. Multiple comparisons for blood lipid profile in rats given corn oil, a physical blend and structured lipids.

\begin{tabular}{|c|c|c|c|c|c|}
\hline \multicolumn{2}{|c|}{ Serum Triacylglycerol } & \multirow{2}{*}{ Mean Difference (I-J) } & \multirow{2}{*}{ Sig. } & \multicolumn{2}{|c|}{ 95\% Confidence Interval } \\
\hline (I) Group & (J) Group & & & Lower Bound & Upper Bound \\
\hline \multirow[t]{2}{*}{ Control } & Structured lipid & $32.84667^{*}$ & 0.002 & 13.0521 & 52.6413 \\
\hline & Physical blend & $25.21500^{*}$ & 0.012 & 5.4204 & 45.0096 \\
\hline \multirow[t]{2}{*}{ Structured lipid } & control & $-32.84667^{*}$ & 0.002 & -52.6413 & -13.0521 \\
\hline & Physical blend & -7.63167 & 0.587 & -27.4263 & 12.1629 \\
\hline \multirow[t]{2}{*}{ Physical blend } & control & $-25.21500^{*}$ & 0.012 & -45.0096 & -5.4204 \\
\hline & Structured lipid & 7.63167 & 0.587 & -12.1629 & 27.4263 \\
\hline \multicolumn{2}{|c|}{ Serum Total Cholesterol } & \multirow{2}{*}{ Mean Difference (I-J) } & \multirow{2}{*}{ Sig. } & \multicolumn{2}{|c|}{ 95\% Confidence Interval } \\
\hline (I) Group & (J) Group & & & Lower Bound & Upper Bound \\
\hline \multirow[t]{2}{*}{ Control } & Structured lipid & $32.84667^{*}$ & 0.002 & 13.0521 & 52.6413 \\
\hline & Physical blend & $25.21500^{*}$ & 0.012 & 5.4204 & 45.0096 \\
\hline \multirow[t]{2}{*}{ Structured lipid } & Control & $-32.84667^{*}$ & 0.002 & -52.6413 & -13.0521 \\
\hline & Physical blend & -7.63167 & 0.587 & -27.4263 & 12.1629 \\
\hline \multirow[t]{2}{*}{ Physical blend } & Control & $-25.21500^{*}$ & 0.012 & -45.0096 & -5.4204 \\
\hline & Structured lipid & 7.63167 & 0.587 & -12.1629 & 27.4263 \\
\hline \multicolumn{2}{|c|}{ HDL-Cholesterol } & \multirow{2}{*}{ Mean Difference (I-J) } & \multirow{2}{*}{ Sig. } & \multicolumn{2}{|c|}{ 95\% Confidence Interval } \\
\hline (I) Group & (J) Group & & & Lower Bound & Lower Bound \\
\hline \multirow[t]{2}{*}{ Control } & Structured lipid & $12.35797^{*}$ & 0.000 & 6.7247 & 17.9912 \\
\hline & Physical blend & $10.12131^{*}$ & 0.001 & 4.4881 & 15.7545 \\
\hline \multirow[t]{2}{*}{ Structured lipid } & Control & $-12.35797^{*}$ & 0.000 & -17.9912 & -6.7247 \\
\hline & Physical blend & -2.23666 & 0.569 & -7.8699 & 3.3966 \\
\hline \multirow[t]{2}{*}{ Physical blend } & Control & $-10.12131^{*}$ & 0.001 & -15.7545 & -4.4881 \\
\hline & Structured lipid & 2.23666 & 0.569 & -3.3966 & 7.8699 \\
\hline \multicolumn{2}{|c|}{ LDL-Cholesterol } & \multirow{2}{*}{ Mean Difference (I-J) } & \multirow{2}{*}{ Sig. } & \multicolumn{2}{|c|}{ 95\% Confidence Interval } \\
\hline (I) Group & (J) Group & & & Lower Bound & Upper Bound \\
\hline \multirow[t]{2}{*}{ Control } & physical blend & $15.82182^{*}$ & 0.000 & 8.0833 & 23.5603 \\
\hline & Structured lipids & 5.62455 & 0.189 & -2.1139 & 13.3630 \\
\hline \multirow[t]{2}{*}{ physical blend } & Control & $-15.82182^{*}$ & 0.000 & -23.5603 & -8.0833 \\
\hline & Structured lipids & $-10.19727^{*}$ & 0.008 & -17.9358 & -2.4588 \\
\hline \multirow[t]{2}{*}{ Structured lipids } & Control & -5.62455 & 0.189 & -13.3630 & 2.1139 \\
\hline & physical blend & $10.19727^{*}$ & 0.008 & 2.4588 & 17.9358 \\
\hline
\end{tabular}

agreed with those of Lee and his colleagues [33]. The effects of SL on serum TC did not differ from those fed PB group, possibly due to the purification steps of the SL, which might result in the esterification of tocopherols to tocopherol esters. Hamam and Shahidi [36] found that tocopherols in the oils were proved to react with carboxylic acids of free fatty acids, therefore leading to the formation of esters of tocopherols. We would expect that formation of tocopheryl esters during the purification steps would affect the cholesterol lowering ability of the SL. 


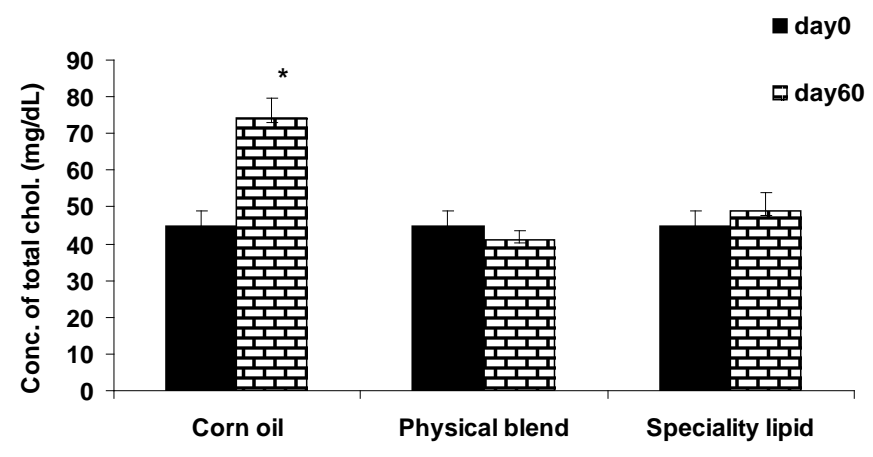

Figure 3. Total cholesterol level (mg/dL) for corn oil, physical blend of fish oil and capric acid and structured lipid composed of fish oil and capric acid at Day zero and after 60 days of treatment. Error bars represent the standard error, ${ }^{*} p$-value $<0.05$ compared to Day zero.

\subsection{Effects of CO, PB, and SL on Serum HDL-c}

Blood lipid analysis for Group 1 (fed with corn oil) and Group 3 (fed with SL) showed significant increase ( $p<$ 0.05) in HDL-c on Day 60 compared to Day 0 (Figure 4). Meantime, animals in Group 2 (fed with a mixture of FO and CA) showed insignificant increase $(p>0.05)$ in HDL-c level (Figure 4). An interesting finding of the study was the significant $(p<0.05)$ higher level of HDL-c in the CO group compared with the other two groups. SL increased HDL-c but this increase was significantly $(p<0.05,95 \%$ CI $=6.72$ to 17.99$)$ lowered than the corresponding level in CO fed animal (Table 1). Furthermore, CO caused higher significant $(p<0.05,95 \%$ $\mathrm{CI}=4.49$ to 15.75) level of HDL-c than those fed SL (Table 1). Results of HDL-c in the reference oil (corn) were inconsistent with other research works [30] [31] [33] [37]. Results of this study indicated that the difference in HDL-c level among rats fed with PB or SL was insignificant $(p<0.05,95 \%$ CI $=-3.39$ to 7.87$)$ (Table 1).

\subsection{Effects of CO, PB, and SL on Serum LDL-c}

Animals in CO and SL groups showed an increase in the level of LDL-c at day sixty of treatment compared to Day zero, but this increase was statistically insignificant $(p>0.05)$ (Figure 5). Whereas, PB significantly $(p<$ 0.05 ) reduced LDL-c level after 60 days of treatment (Figure 5). These results disagreed with those of Dauqan et al. [31] who found that feeding rats with CO for 8 weeks caused a significant decrease $(p<0.05)$ in LDL-c level. CO could be considered as LDL-c lowering agent when taking into consideration the high level of PUSFA ( 59\%), MUSFA (24\%) and low level (13\%) of SFA, but results of this study showed that CO had no effect on LDL-c. Thus, further studies should be conducted to investigate these results. On the other hand, at 8 weeks, the level of LDL-c is considerably $(p<0.05$ ) decreased in animals fed with a mixture of FO and CA (Figure 5). Furthermore, this decrease was significant $(p<0.05$; 95\% CI $=-17.93$ to -2.46$)$ in rats fed a PB compared to those fed SL (Table 1). Lee et al. [33] found different results when they fed rats different types of dietary lipids (SL vs PB of soybean oil with caprylic acid). The difference in LDL-c level among rats fed SL and those fed CO was insignificant ( $p>0.05$; 95\% CI $=-2.46$ to 13.36) (Table 1 ), but upon comparison with those fed PB the difference was statistically significant $(p<0.05$; 95\% CI $=8.08$ to 23.56) (Table 1 ). One possible reason for such a decrease in LDL-c could be due to stimulation of breakdown of LDL-c by high content of PUSFA of the PB. Theoretically, the order of reduction of LDL-c of three diets should be in the order SL > PB > CO when structural specificity and fatty acid proportions of PUSFA/MUSFA/SFA are taken into consideration. Although, the physical blend diet has less structural specificity and more SFA, it resulted in the highest significant $(p<$ 0.05 ) decrease in LDL-c level compared to those fed either CO or SL. Reasons behind significant decrease caused by the physical blend remain unclear. Thus, further work is needed to investigate these results.

\subsection{Body Weight Test}

Percent change in body weight of normal rats fed CO, PB and SL for 8 weeks is shown in Figure 6. The weight of animals was increasing with time in animals fed CO, while it was decreasing with time for animals fed PB 


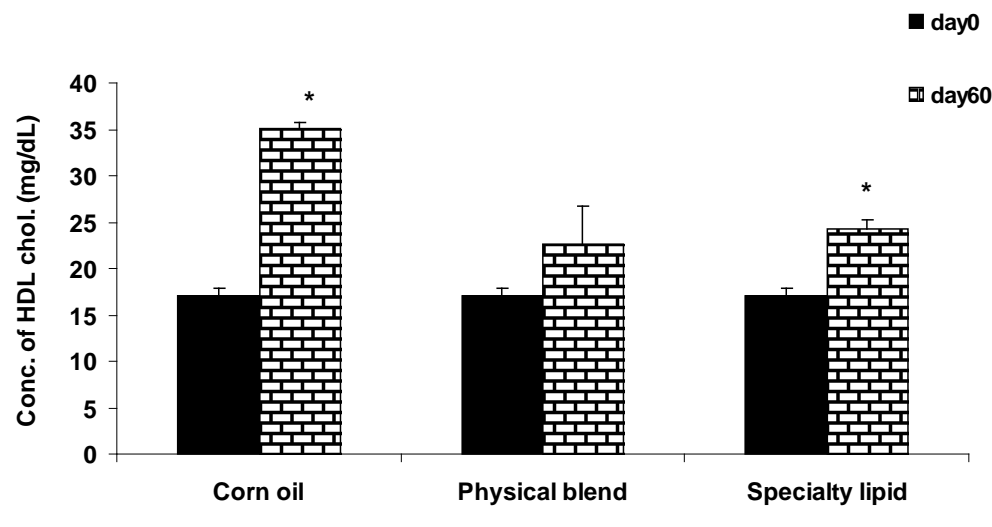

Figure 4. HDL-C concentration (mg/dL) for corn oil, physical blend of fish oil and capric acid and structured lipid composed of fish oil and capric acid at Day zero and after 60 days of treatment. Error bars represent the standard error, * $p$-value $<0.05$ compared to day zero.

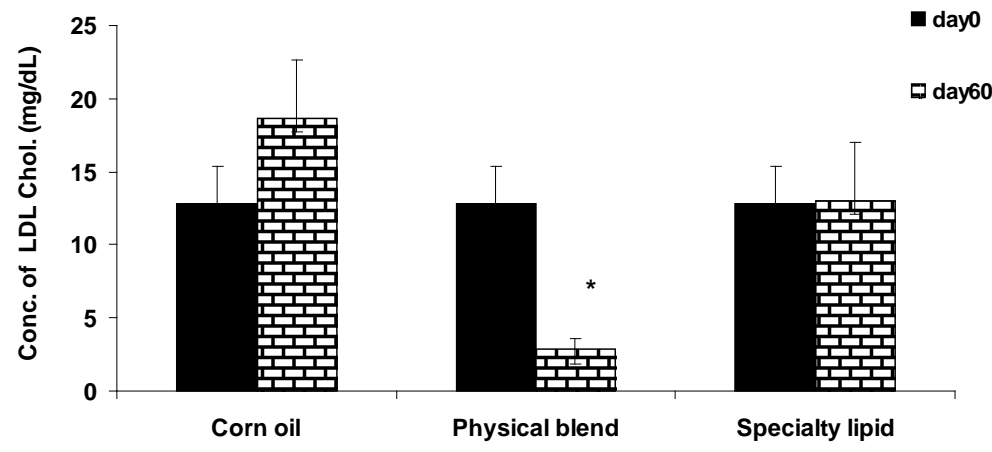

Figure 5. LDL-C level in (mg/dL) for corn oil, physical blend of fish oil and capric acid and structured lipid composed of fish oil and capric acid at Day zero and after 60 days of treatment. Error bars represent the standard error, * $p$-value $<0.05$ compared to Day zero.

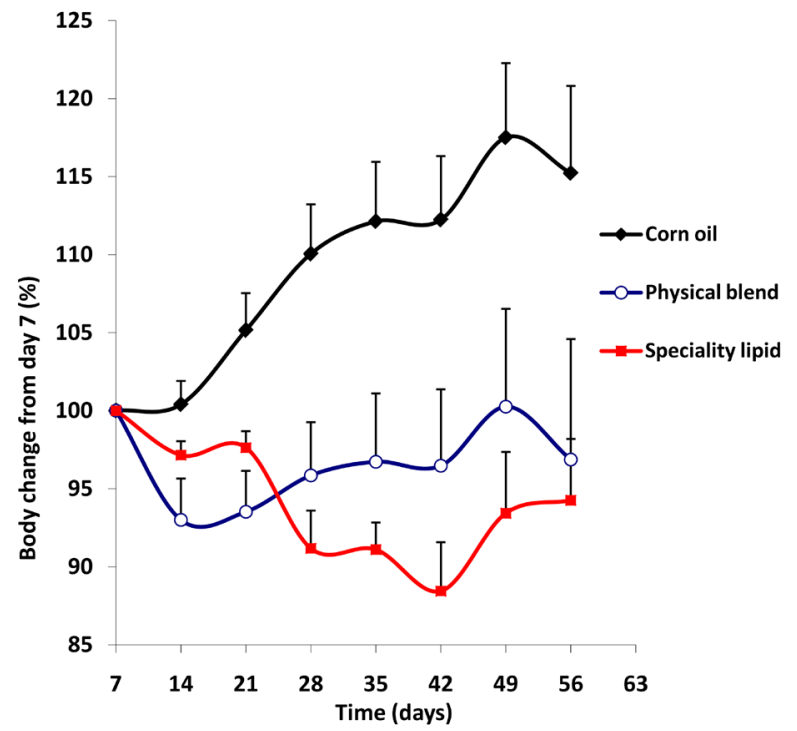

Figure 6. Percent change in body weight of normal rats fed corn oil, physical blend of fish oil and capric acid and structured lipid composed of fish oil and capric acid for 8 weeks. 
Table 2. Multiple comparisons in percent change in body mass of rats given corn oil, a physical blend and structured lipids.

\begin{tabular}{cccccc}
\hline & & Mean Difference & & \multicolumn{2}{c}{$95 \%$ Confidence Interval } \\
\cline { 5 - 6 }$(\mathrm{I})$ Group & (J) Group & & Sig. & Lower Bound & Upper Bound \\
\hline Control & Structured lipid & $17.11375^{*}$ & 0.000 & 10.6183 & 23.6092 \\
& Physical blend & $14.93625^{*}$ & 0.000 & 8.4408 & 21.4317 \\
\multirow{2}{*}{ Structured lipid } & Control & $-17.11375^{*}$ & 0.000 & -23.6092 & -10.6183 \\
& Physical blend & -2.17750 & 0.680 & -8.6729 & 4.3179 \\
Physical blend & Control & $-14.93625^{*}$ & 0.000 & -21.4317 & -8.4408 \\
& Structured lipid & 2.17750 & 0.680 & -4.3179 & 8.6729 \\
\hline
\end{tabular}

and SL. The difference between either SL or PB with CO was significant $(p<0.05)$ after 8 weeks of treatment. However, the difference between SL and PB was insignificant $(p>0.05)$ at all time intervals. Furthermore, statistical analysis was performed using the Tukey test, and results lend further support to the significant difference between animals fed SL and those fed CO $(P<0.05) 95 \%$ confidence interval $(\mathrm{CI}=10.62-23.61)$ was also significant (Table 2). The same trend was noted upon comparing animals given $\mathrm{PB}$ and those given $\mathrm{CO}(p<$ 0.05 ) $95 \%$ confidence interval ( $\mathrm{CI}=-21.43$ to -8.44 ) (Table 2). Although, the percent body change in animals fed SL was lower than those given PB (mean difference between SL and PB $=-2.1775$ ), the difference was insignificant $(p>0.05)$ and 95\% confidence interval (CI $=-4.32-8.67)$ (Table 2). This study suggested that increased body weight in rats fed CO could be attributed to high uptake of exogenous fats or oils and subsequent deposition of them in the body. Another possible reason for such an increase could be due to a decrease in catabolism of fats or oils. Increased body weight in CO fed animals could be also explained through enhanced lipogensis. None the less, these assumptions should be tested in a future study. Specialty lipids produced in this study contained a good amount of MUSFA ( 20\%) and PUSFA (32\%), this combination of MUSAF and PUSFA could promote a better TAG clearance and, thus, no subsequent deposition of fats or oils would occur. Moreover, SL contained a good level of MCFA (C10:0) which display unique structural and physiological properties. These MCFA are transported quickly to liver for fast energy release and their affinity to accumulate as body fat is much lower than LCFA.

\section{Conclusion}

This study produced large amount of specialty lipids based on fish oil and a medium-chain fatty acid (C10:0) using a novel single bed bioreactor packed with a specific sn-1,3 lipase. SL improved blood lipid profile as it caused a significant decrease in TAG and considerable increase in HDL-c after 60 days of treatment, but SL had no effects on TC and LDL-c. Moreover, SL caused a significant decrease in the percent body change compared to the reference oil (CO). The physical mixture of FO and CA had positive impacts on blood lipid chemistry as it caused a considerable decrease in TAG and LDL-c, but had no effects on TC and HDL-c. Effects of corn oil on blood lipid chemistry were varying. For example, it caused a significant decrease in TAG and a substantial increase in HDL-c. On the other hand, CO increased TC and had no effects on LDL-c. This study was conducted on normal healthy rats as a preliminary work to investigate the effect of SL on blood lipid profile compared to a PB and CO. However, it is recommended to carry out a further study on hyperlipidemic rat model to show the biochemical action of such material on animals with a metabolic disorder.

\section{References}

[1] Smith, G.D. (2001) Is It Time to Call It a Day? International Journal of Epidemiology, 30, 1-11. http://dx.doi.org/10.1093/ije/30.1.1

[2] Manios, Y., Moschandreas, J., Hatzis, C. and Kafatos, A. (2002) Health and Nutrition Education in Primary Schools of Crete: Changes in Chronic Disease Risk Factors Following a 6-Year Intervention Programme. British Journal of Nutrition, 88, 315-324.

[3] Al Balla, S.R., Sekait, M. and Balla, M. (1993) Causes of Morbidity in the Elderly Population of Saudi Arabia. Journal of Tropical Medicine and Hygiene, 96, 157-162. 
[4] Kumosani, T.A., Alama, M.N. and Iyer, A.P. (2011) Cardiovascular Diseases in Saudi Arabia. Prime Research on Medicine, 1, 1-6.

[5] Al Balla, S.R., Bamgboye, E.A., Al Balla, S.R., Al Sekait, M. and Al Rasheed, R. (1993) Pattern of Adult Admission into Medical Wards of King Khalid University Hospital, Riyadh (1985-1990). Saudi Medical Journal, 13, 8-13.

[6] Sulieman, N., Al-Shehri, Z.A.S., Salama, M.M. and Hassan, Y.H. (2004) Prevalence of Hyperlipidemia among Saudi School Children in Riyadh. Annals of Saudi Medicine, 24, 6-8.

[7] Al-Nuaim, A.R., Al-Rubeaan, K., Al-Mazroub, Y., Al-Attas, O. and Al-Daghari, N. (1996) Prevalence of Hypercholesterolemia in Saudi Arabia, Epidemiological Study. International Journal of Cardiology, 54, 41-49. http://dx.doi.org/10.1016/0167-5273(96)02555-7

[8] Al-Nuaim, A.R., Al-Rubeaan, K., Al-Mazroub, Y., Al-Attas, O. and Al-Daghari, N. (1997) Serum Total and Fractionated Cholesterol Distribution and Prevalence of Hypercholesterolemia in Urban and Rural Communities in Saudi Arabia International Journal of Cardiology, 58, 141-149.

[9] Gunstone, F.D. and Padley, F.B., Eds. (1997) Lipid Technologies and Applications. Marcel Dekker Inc., New York, 834.

[10] Akoh, C.C. (2002) Structured Lipids. In: Akoh, C.C. and Min, D.B., Eds., Food Lipids, Marcel Dekker Inc., New York, 877-908. http://dx.doi.org/10.1201/9780203908815.ch28

[11] Hamam, F. and Budge, S. (2010) Structured and Specialty Lipids in Continuous Packed Column Reactors: Comparison of Production Using One and Two Enzyme Beds. Journal of the American Oil Chemists' Society, 87, 385-394.

[12] Canada, O.N. (2013) Omega-3 Overview: Ride the Mutli-Bllion-Dollar Wave. Nova Scotia.

[13] Breslow, J.L. (2006) n-3 Fatty Acids and Cardiovascular Disease. American Journal of Clinical Nutrition, 83, 1477S1482S.

[14] Von Schacky, C. (2007) Omega-3 Fatty Acids and Cardiovascular Disease. Current Opinion in Clinical Nutrition and Metabolic Care, 10, 129-135. http://dx.doi.org/10.1097/MCO.0b013e3280127af0

[15] Swanson, D., Block, R. and Mousa, S.A. (2012) Omega-3 Fatty Acids EPA and DHA: Health Benefits throughout Life. Advances in Nutrition, 3, 1-7.

[16] Nicholson, T., Khademi, H. and Moghadasian, M.H. (2013) The Role of Marine n-3 Fatty Acids in Improving Cardiovascular Health: A Review. Food \& Function, 4, 357-365. http://dx.doi.org/10.1039/c2fo30235g

[17] Simopoulos, A.P. (2002) Omega-3 Fatty Acids in Inflammation and Autoimmune Diseases. Journal of the American College of Nutrition, 21, 495-505. http://dx.doi.org/10.1080/07315724.2002.10719248

[18] Hartweg, J., Montori, P.R., Dinneen, S.F., Ahawn, N. and Farmer, A.J. (2008) Omega-3 Polyunsaturated Fatty Acids (PUFA) for Type 2 Diabetes Mellitus. Cochrane Database of Systematic Reviews, 1, 42-45.

[19] Atar, M.J.H., Hajianfar, H. and Bahonar, A. (2012) The Effects of Omega-3 on Blood Pressure and the Relationship between Serum Visfatin Level and Blood Pressure in Patients with Type II Diabetes. Atherosclerosis Journal, 8, 27-31.

[20] Mori, T.A. (2006) Omega-3 Fatty Acids and Hypertension in Humans. Clinical and Experimental Pharmacology and Physiology, 33, 842-846. http://dx.doi.org/10.1111/j.1440-1681.2006.04451.x

[21] Fassett, R.G., Peake, J.M. and Coombes, J.S. (2010) Omega-3 Polyunsaturated Fatty Acids in the Treatment of Kidney Disease. American Journal of Kidney Diseases, 56, 728-742. http://dx.doi.org/10.1053/j.ajkd.2010.03.009

[22] Harris, W.S. and Von Schacky, C. (2004) The Omega-3 Index: A New Risk Factor for Death from Coronary Heart Disease. Preventive Medicine, 39, 212-220. http://dx.doi.org/10.1016/j.ypmed.2004.02.030

[23] Heird, W.C., Grundy, S.M. and Hubbard, V.S. (1986) Structured Lipids and Their Use in Clinical Nutrition. American Journal of Clinical Nutrition, 43, 320-324.

[24] Lee, T.W. and Hastilow, C.I. (1999) Quantitative Determination of Triacylglycerol Profile of Structured Lipid by Capillary Supercritical Fluid Chromatography and High-Temperature Gas Chromatography. Journal of the American Oil Chemists' Society, 76, 1405-1413. http://dx.doi.org/10.1007/s11746-999-0176-2

[25] Lee, K.T. and Akoh, C.C. (1998) Structured Lipids: Synthesis and Application. Food Reviews International, $14,17-34$. http://dx.doi.org/10.1080/87559129809541148

[26] Bell, S.J., Mascioli, E.A., Bistrian, B.R., Babayan, V.K. and Blackburn, G.L. (1991) Alternative Lipid Sources for Enteral and Parenteral Nutrition: Long- and Medium-Chain Triglycerides, Structured Triglycerides, and Fish Oils. Journal of the American Dietetic Association, 91, 74-78.

[27] Friedewald, W.T., Levy, R.I. and Fredrickson, D.S. (1972) Estimation of the Concentration of Low-Density Lipoprotein Cholesterol in Plasma, without Use of the Preparative Ultracentrifuge. Clinical Chemistry, 18, 499-502.

[28] Ng, T.K.W., Hassan, K., Lim, J.B., Lye, M.S. and Ishak, R. (1991) Nonhypercholesterolemic Effects of a Palm-Oil Diet in Malaysian Volunteers. American Journal of Clinical Nutrition, 53, 1015S-1020S. 
[29] Nalbone, G., Leonardi, J., Termine, E., Portugal, H., Lechene, P., Pauli, A.M. and Lafont, H. (1989) Effects of Fish Oil, Corn Oil and Lard Diets on Lipid Peroxidation Status and Glutathione Peroxidase Activities in Rat Heart. Lipids, 24, 179-186. http://dx.doi.org/10.1007/BF02535232

[30] Ismael, O.E., Hashish, E.A. and Ali, H.A. (2014) Lipid Profile and Lipogensis Following Corn Oil, Truffle Oil or Wheat Germ Oil Administration in Albino Rat. Global Veterinaria, 12, 461-469.

[31] Dauqan, E., Sani, H.A., Abdullah, A. and Kasim, Z.M. (2011) Effect of Different Vegetable Oils (Red Palm Olein, Palm Olein, Corn Oil and Coconut Oil) on Lipid Profile in Rat. Food and Nutrition Sciences, 2, 253-258. http://dx.doi.org/10.4236/fns.2011.24036

[32] Dupont, J., White, P.J., Carpenter, M.P., Schaefer, E.J., Meydani, S.N., Elson, C.E., Woods, M. and Gorbach, S.L. (1990) Food Uses and Health Effects of Corn Oil. Journal of the American College of Nutrition, 9, 438-470. http://dx.doi.org/10.1080/07315724.1990.10720403

[33] Lee, K.T., Akoh, C.C., Flatt, W.P. and Lee, J.H. (2000) Nutritional Effects of Enzymatically Modified Soybean Oil with Caprylic Acid versus Physical Mixture Analogue in Obese Zucker Rats. Journal of Agricultural and Food Chemistry, 48, 5696-5701. http://dx.doi.org/10.1021/jf000072+

[34] Rezq, A.A., Labib, F.A. and Attia, A.E.M. (2010) Effect of Some Dietary Oils and Fats on Serum Lipid Profile, Calcium Absorption and Bone Mineralization in Mice. Pakistan Journal of Nutrition, 9, 643-650. http://dx.doi.org/10.3923/pjn.2010.643.650

[35] Karaji-Bani, M., Montazeri, F. and Hashemi, M. (2006) Effect of Palm Oil on Serum Lipid Profile in Rats. Pakistan Journal of Nutrition, 5, 234-236. http://dx.doi.org/10.3923/pjn.2006.234.236

[36] Shahidi, F. and Hamam, F. (2006) Acidolysis Reactions Lead to Esterification of Endogenous Tocopherols and Compromised Oxidative Stability of Modified Oils. Journal of Agricultural and Food Chemistry, 54, 7319-7323. http://dx.doi.org/10.1021/jf061730e

[37] Seneviratne, K.N., Kotuwegedara, R.T. and Ekanayake, S. (2011) Serum Cholesterol and Triglyceride Levels of Rats Fed with Consumer Selected Coconut Oil Blends. International Food Research Journal, 18, 1303-1308. 